Revue d'histoire de l'Amérique française

REYUE D.HISTOIRE DE L'AMÉRIQUE FRANÇAISE

\title{
L'Histoire et la recherche du sens
}

Discours de réception à l'Académie canadienne-française

\section{Jean-Pierre Wallot}

Volume 37, numéro 4, mars 1984

URI : https://id.erudit.org/iderudit/304205ar

DOI : https://doi.org/10.7202/304205ar

Aller au sommaire du numéro

Éditeur(s)

Institut d'histoire de l'Amérique française

ISSN

0035-2357 (imprimé)

1492-1383 (numérique)

Découvrir la revue

Citer ce document

Wallot, J.-P. (1984). L'Histoire et la recherche du sens : discours de réception à l'Académie canadienne-française. Revue d'histoire de l'Amérique française, 37(4), 533-542. https://doi.org/10.7202/304205ar d'utilisation que vous pouvez consulter en ligne.

https://apropos.erudit.org/fr/usagers/politique-dutilisation/ 


\title{
L'HISTOIRE ET LA RECHERCHE DU SENS Discours de réception à l'Académie canadienne-française
}

\author{
JEAN-PIERRE WALLOT
}

"Suis-je oublié déjà comme ces vieilles ancres, Abordé par le soir le quai devient plus triste, Et ma vie est lassée de sa faim inutile...»

(Pablo NERUDA)

Monsieur le président, Monsieur Brunet, qui m'avez fait l'honneur de me présenter, Membres de l'Académie, Mesdames, Messieurs,

Ce cri du coeur de Néruda, par delà son universalité, exprime bien l'angoisse secrète de l'historien. La discipline historique, en effet, ne consiste pas à construire des édifices bien planifiés, étage après étage, selon une logique linéaire et un temps étale, étrangers au déferlement touffu, souvent imprévisible et spasmodique de l'«histoire». Elle souffre - et fait souffrir - de ce que Paul Leuillot a appelé «le haut taux de mortalité historique»: mortalité précoce des hypothèses, des éclairages, des problématiques, bref des questions et des réponses qui deviennent si rapidement caduques, avec chaque cohorte de spécialistes, hormis pour un aspect essentiel - leur interpellation de notre conscience de la condition humaine; mortalité qui, plus superficiellement, s'accorde bien avec une culture de raccourcis et de bonds caractéristiques d'une société en rapide évolution; mortalité enfin qui, dans cette discipline plus que dans d'autres, dans ce pays plus qu'en d'autres, se traduit par un dénigrement systématique des devanciers pour mieux rehausser les modestes réalisations des successeurs. Et pourtant, paradoxalement, c'est cette mortalité même qui appelle au défi, au dépassement dans une science qui est, selon l'expression de Lucien Febvre, une «science de la vie... qu'elle prétend reconstituer».

Mais est-ce bien la vie qu'elle recrée? N'exprimerait-elle que les architectures rétrospectives, les rétrodictions de nos hantises présentes? $\mathrm{Ou}$, comme le pense Khalil Gibran, dans Le Prophète, nous confronterait-elle au temps, donc à «l'intemporel» qui nous habite tous et qui «est conscient de l'intemporalité de la vie, et sait qu'aujourd'hui n'est que le souvenir d'hier et demain le rêve d'aujourd'hui... Et qu'aujour- 
d'hui embrasse le passé avec souvenir et le futur avec aspiration». Jouer aux futuribles du passé, c'est là un métier de haute voltige qu'ont pratiqué nombre de membres passés et présents de cette Académie qui me fait l'honneur de m'accueillir. Aussi, convient-il d'esquisser l'apport de quelques-uns d'entre eux. Puis, plutôt que de paraphraser deux articles récents que j'ai consacrés à ma vision de l'histoire ${ }^{1}$, j'évoquerai un peu comme en un kaléidoscope, quelques moments-clé, quelques questions et quelques postulats qui m'ont aiguillé vers l'histoire et qui conservent à mes yeux sa fascination à une discipline dont les apports sous-tendent toute réflexion sur l'homme et la société. Car c'est par l'universel que je suis venu à l'histoire, et c'est vers lui qu'elle me refoule.

\section{I}

Le premier qui m'a initié à l'opération essentielle de l'épluchage des sources d'archives et de la formulation de questions cohérentes à leur poser, sans lesquelles on ne saurait débusquer de réponses satisfaisantes, c'est Michel Brunet. Je le remercie de m'avoir présenté à l'Académie, ce soir, de façon à la fois affectueuse, humoristique et critique. C'est lui également qui m'a ouvert la porte à une carrière universitaire. Or, particulièrement en cette décennie 1950-1960, alors qu'une bonne partie de l'intelligentsia universitaire avait abdiqué devant sa responsabilité de définir des intérêts collectifs propres à notre «petit peuple» (Groulx) pour promouvoir une intégration «lucide» aux idées et aux dollars de la majorité canadian, sa combativité, sa ténacité, sa perspicacité à dégager des tendances essentielles du fatras des événements contemporains ${ }^{2}$, sa disponibilité, enfin, auprès des étudiants et des collègues, toutes ces qualités, parmi d'autres, lui ont valu une admiration continue et méritée, ainsi que des inimitiés tenaces et souvent mesquines.

Quant au regretté Guy Frégault, la rigueur croissante de ses travaux, la clarté de son discours, à la fois élégant et mordant, sa culture continuellement nourrie aux meilleures sources, ne pouvaient que nous éblouir et nous stimuler. Trait caractéristique, la meilleure note qu'il m'ait décernée pour un travail surplombait ce commentaire concis: «presque pas un mot de trop»!

Maurice Séguin, aux idées plus incisives et plus critiques, alliait l'ironie à la provocation intellectuelle. Il nous confrontait, pour la première fois peut-être en histoire du Canada-Québec, à un système de pensée organisé, charpenté, à ce qu'on appelle aujourd'hui un modèle

\footnotetext{
J.-P. Wallot, «L'histoire, science de la vie» in Présentations [...] à la Société royale du Canada, 1979-1980 (Ottawa, 1980): 33-47; «De l'indiscipline historique et de la régulation des passés au présent», Liberté, 147 (juin 1983): 57-62.

2 On trouvera plusieurs textes de M. Brunet qui datent de cette période dans Canadians et Canadiens (Montréal, 1954).
} 
sociétal $^{3}$. Quant au Père Antonin Papillon, son érudition ahurissante fusionnait, dans un raccourci saisissant, un fait en apparence banal d'histoire romaine ou du Moyen Âge et un événement survenu à l'un ou l'autre de nos parents dont nous ignorions parfois jusqu'à l'existence.

Ce n'est que très tard que je connus personnellement - et assez peu - mon prédécesseur à ce siège de l'Académie, le regretté Robert Rumilly. Ce dernier a planté une forêt irremplaçable dans notre historiographie, une forêt si dense que ses contemporains et successeurs ont pu la piller allègrement sans vraiment la dénuder. Chez Rumilly, comme l'a souligné récemment Pierre Trépanier ${ }^{4}$, le polémiste politique et le penseur de droite ont débordé sur l'historien et vice-versa. Il a semé quelques tempêtes et récolté la foudre de collègues. Il reste que cet auteur d'une prodigieuse masse d'études, de biographies, de pamphlets, a été le premier à baliser notre histoire après la Confédération. Sa monumentale Histoire de la province de Québec, qui comprend 41 tomes publiés entre 1940 et 1969 , dévide la chronique socio-politique du passé récent du Québec et a irrigué presque tous les enseignements et les études en cette matière depuis la fin des années 1940. Les critiques fusèrent drues, parfois féroces, contre les premiers volumes: contrairement à Groulx, qui s'intéressait au «tableau», à la «synthèse», à l'«architecture» de l'évolution historique du Canada français, donc qui concevait l'histoire à la moderne, c'est-à-dire comme une science explicative du passé ${ }^{5}$, Rumilly décrivait, racontait, étalait documents et extraits d'articles, montait une chronique, une série de séquences et de plans dont le cadre d'interprétation paraissait inexistant ou savamment camouflé derrière ce que d'aucuns appellent «l'objectivité» ou le détachement de l'historien. Il est curieux, aujourd'hui, de rappeler que Rumilly ne considérait pas Groulx comme un historien pour cela même qui passe de nos jours pour l'essence de l'histoire: le choix conscient des «faits», des matériaux de la reconstruction historique, à partir d'un plan, d'une grille explicitée de questions, de façon à ce qu'émerge une explication, une compréhension, une plus grande conscience du passé probable.

À mesure que l'Histoire de la province de Québec se déroulait, pour ainsi dire, de volume en volume, la plupart des critiques, même les plus sévères, en sont venus à rendre les armes et à reconnaître l'apport substantiel et original de Rumilly. Plus tard, malgré son âge très avancé, ce dernier a réussi à publier une volumineuse histoire de Duplessis, un Papineau revu et corrigé, de nombreuses histoires de

\footnotetext{
réal, 1965).

Voir ses «Normes» polycopiées sous le titre Sociologie du national (Université de Mont-

4 P. Trépanier, «Robert Rumilly, historien engagé», L'Action nationale, 73, 1 (septembre 1983): 9-40.

J.-P. Wallot, «Groulx historiographe», RHAF, 32 (décembre 1978): 407-433.
} 
villes et de paroisses, dont l'une controversée, sur Montréal, une Histoire des Acadiens considérablement augmentée, etc.

Une énigme pourtant demeure; et elle captive d'autant plus qu'elle se noue au coeur même du métier d'historien: c'est le cloisonnement apparent et très marqué entre Rumilly l'historien et Rumilly le polémiste; entre le récit ou la chronique, d'une part, et d'autre part, les opinions politiques et sociales de ce nationaliste maurassien, défenseur de l'autonomie du Québec vu comme l'État national des Canadiens français. À l'instar de Groulx, il adhère à l'idéal d' «un Québec autonome dans un Canada indépendant»; mais contrairement au premier, il faut lire ses écrits politiques et polémiques pour en inférer clairement cette vision qui, estompée, court en filigrane dans ses études historiques. Comment l'homme pouvait-il ainsi départager ses opinions partisanes de son travail d'historien, son idéologie de sa collecte des faits qu'il jugeait significatifs et de leur organisation en un discours-récit?

Une relecture attentive de l'oeuvre montre que par ses choix, ses omissions, ses préférences et, plus rarement, des commentaires explicites, Rumilly n'a pas échappé aux règles de son métier: c'est-à-dire établir ce qui s'est passé et l'expliquer par rapport à des pôles de référence qui constituent, en quelque sorte, le treillis de questions-réponses émanant des attentes et des inquiétudes des contemporains.

«La culture, a écrit André Malraux, est faite de tout ce qui permet à l'homme de maintenir l'image idéale de lui-même qu'il a héritée.» Et le rôle de l'historien, comme celui de tout intellectuel, serait donc de «tenter de donner conscience [aux] hommes de la grandeur qu'ils ignorent en eux», c'est-à-dire de cet héritage; plus spécifiquement, de démêler et de tisser les filons de la personnalité collective, acquise à travers les épreuves du temps, personnalité qui, par l'illumination du tableau et du récit (de la structure et de la conjoncture), se saisit consciemment et se projette dans l'avant et l'après. "Consciemment», ai-je dit, car l'inconscient, c'est-à-dire le subi, l'imposé, peut-il être l'histoire? Ne faut-il pas un dépassement, un défi au destin par l'action consciente et la création? Mais «que la chose nous plaise ou ne nous plaise pas, commentait Groulx, nous sommes une résultante, un aboutissement d'histoire. Dans les vivants que nous sommes, survivent combien de morts qui nous expliquent ${ }^{6}$.

Le «tableau» des influences serait incomplet sans de nombreuses autres mentions ${ }^{7}$. Je me contenterai de rappeler l'apport essentiel de ma femme, dont la dialectique naturelle et la curiosité enthousiaste ont fait cheminer bien des recherches et des interprétations; celui également de l'économiste Gilles Paquet, toujours à l'affût du rationnel, du généra-

\footnotetext{
6 L. Groulx, «Un Institut d'histoire», RHAF, 2 (1948-1949): 472-473.

Voir mon texte «L"histoire, science de la vie».
} 
lisable, de l'interdisciplinaire, mais surtout de la lucidité. Combien de préjugés ai-je dû abandonner après qu'il m'eut forcé à en prendre conscience. À eux, à tant d'autres collègues, amis, et de générations d'étudiants, je suis redevable.

\section{II}

Pourquoi et comment vient-on à l'histoire? Il n'y a certes pas de voie royale et unique. Dans mon cas personnel, l'histoire commence peut-être par le vertige du vide originel, puis par les nappes décantées du brouillard plus ou moins opaque des souvenirs lointains, des murmures, du ressenti, du non-dit. Est-ce la lave chaude de l'imagination de l'enfance qui s'enflamme aux contes des mousquetaires et aux récits illustrés de la Grande Guerre dans la bibliothèque de mes parents et celle de ma grand-mère; ou qui cascade, à l'école, sur la piste des Dollard, des d'Iberville, des miliciens canadiens harcelant les grosses armées d'invasion britanniques, des obscurs résistants et résistantes qui ont maintenu leur culture et la cohésion de leur peuple, des Bédard, des Papineau, des Lafontaine, des Cartier, de tous ces chefs de minoritaires qui se sont battus contre plus forts et plus riches? Est-ce le pouls trop rapide et le serrement à la gorge qui précèdent les combats fréquents (et plutôt symboliques) entre petits «Canayens» et petits «English» qui patrouillent des trottoirs différents, dans une petite ville ouvrière de province appelée Valleyfield? Est-ce le culte familial pour le roi Albert de Belgique qui s'est tué en escaladant une montagne, ou le culte de l'autre moitié de la famille pour son long passé, ses ancêtres et mon grand-père maternel, notaire et un des premiers maires de Lac Mégantic, dont ma mère vient de me donner un souvenir irremplaçable: son permis de pratique du notariat, daté du 11 juin 1883 , soit d'il y a cent ans? Est-ce l'arbre gigantesque de cette famille de seize enfants, dont des rameaux ont pris souche même aux États-Unis, à l'image d'une diaspora très répandue?

Peu m'aurait-il importé d'ailleurs de savoir que Dollard, lui aussi, convoitait des fourrures, que d'Iberville correspond presque au portrait d'un marchand-pirate, que les Canadiens ont longtemps tenu en échec les Britanniques plus nombreux par la terreur d'une guérilla cruelle. Peu m'aurait-il importé de savoir que les élites canadiennes-françaises ont dû pactiser et ont su tirer leur épingle du jeu — une si petite épingle, un si petit jeu! Peu m'aurait-il importé de savoir que la vraie famille et les vrais amis sont ceux du choix assumé, du coeur, pas nécessairement ceux du sang, de l'ethnie ou de l'occupation.

L'histoire se poursuit peut-être par la fierté et la rancoeur qui bouillonnnent quand on découvre que leurs pères ne parlent qu'en anglais à nos pères; que l'armée n'a qu'une langue qui claque, chaque jour, sur «le terrain de parade»; que l'avalanche du quotidien sature la rétine et 
l'esprit du journaliste (que j'étais) et le pousse à mieux capter et cadrer ces images où s'entrechoquent l'individuel et le social, le local, le national et l'international, le matériel et le spirituel.

Plus tard, est-ce le poudroiement fébrile des colonnes de lumière et des éclats de voix qui se répercutent entre les baies vitrées et les briques jaunes-sales de la cafétéria «Chez Valère», là où à l'heure du café, au-dessus d'un liquide douteux et fumant, les maîtres s'escriment avec férocité et respect, tirant de ces corps à corps de l'esprit un plaisir intense que nous ne pouvions partager qu'en spectateurs: en témoins passionnés, mais encore gênés et insuffisamment aguerris pour se mesurer aux Brunet, aux Séguin et aux Frégault au sommet de leur art et de leur éloquence. Ce cercle s'agrandissait d'ailleurs, à l'occasion, de jouteurs redoutables, tels Pierre Trudeau, Jean Beetz, Paul Lacoste et d'autres qui ont ensuite laissé leur «cicatrice» sur la carte de notre histoire.

Et puis, aurais-je pu étouffer l'aspiration profonde que nourrit chaque être humain à la durée et à la signification: vivre longtemps; se prolonger dans une progéniture; constituer des patrimoines physiques et culturels à transmettre aux générations suivantes; récolter une certaine renommée, laisser des traces de son passage par une intervention dans le cours de l'histoire, qu'elle s'incarne dans l'action, dans la puissance politique ou économique, dans une oeuvre philosophique, littéraire ou artistique. Et pour plusieurs, ce sens dépasse l'individu pour revêtir une dimension collective de nature sociale, nationale, intellectuelle, religieuse, etc.

Il y a aussi chez les hommes, du moins chez ceux qui descendent des vaincus, une admiration obscure et instinctive, souvent refoulée derrière un mépris de façade, à l'endroit des petits piétinés qui ont su garder ou se forger une dignité. Certes, la promotion sociale et les contacts transculturels peuvent atténuer ce sentiment, voire nourrir une attitude inverse d'intégration volontaire à d'autres collectivités plus réussies, plus achevées. Pourtant le manteau hérité du passé et d'une culture, les traditions, même oubliées, ont cette curieuse tendance à resurgir à tout moment, comme des échos d'un passé qui aurait pu enchaîner des présents et, qui sait, des futurs autres.

L'histoire est porteuse de sens, de signification. D'où naît cet attrait irrésistible des orphelins pour leurs racines cachées? L'homme ne succombe-t-il pas à une soif inextinguible de connaître, de comprendre son passé, donc de le posséder pour l'exorciser, l'assumer, comme pour en gommer les ignominies obscures ou pour conjurer le temps. Pourquoi tant d'invididus se soumettent-ils à des psychanalyses, formelles ou informelles, sinon pour rattraper les fils épars et cachés de la trame de leur personnalité et pour mieux saisir le cours d'une destinée qui leur échappe? Par analogie, on peut soutenir que les hommes ont besoin de 
savoir d'où ils sourdent, non seulement comme individus mais comme groupes organisés qui se sont échafaudés peu à peu, au cours des siècles, de telle façon plutôt que de telle autre, à tel moment et en tel lieu plutôt qu'en d'autres. Ils veulent faire émerger, au niveau de la conscience, la façon dont collectivement, ils se sont institués en un réseau spécifique de symboles et de relations qui constitue l'architecture de leur société. C'est la quête essentielle de la connaissance de soi, de sa culture, de sa signification.

Pour Toynbee, «la société est le réseau complet des relations entre les êtres humains» 8 : relations d'ordre géographique, démographique, économique, social, politique, idéologique, esthétique - ensemble de relations qui interagissent et se combinent en un fondu qui constitue la trame du récit historique. Faut-il alors s'étonner que l'histoire serve en quelque sorte de discipline-mère, de discipline fiduciaire à la plupart des autres sciences humaines qui viennent y quérir un assortiment diversifié de «faits» pour maçonner, démontrer ou infirmer leurs théories et leurs postulats, les soumettre à la «falsification». Le passé est à chaque groupe comme une mère mythique qui livre un sens à la trajectoire suivie et à orienter. L'histoire est aux autres sciences humaines comme une source de significations, mais aussi d'énigmes. Même si elle est souvent écartelée entre «l'histoire-en-miettes», très empirique, et la fuite dans la métaphysique ou la méthodologie, il n'en demeure pas moins, selon l'heureuse expression de Denis Richet, que «la passion de l'histoire est celle d'une totalité, d'une globalité»' ${ }^{9}$. Son maître, Fernand Braudel, n'avait-il pas énoncé que «seuls comptent les ensembles» ${ }^{10}$.

Si toute réalité s'incarne dans des dimensions spatio-temporelles, le temps n'a pas la même signification pour les uns et les autres. Fautil évoquer en exemple l'échelle presque interminable et pourtant le spasme presque unifié des physiciens - la relativité espace-tempsénergie - , pour qui des «bigs bangs» scandent la respiration de l'univers? Ou rappeler la notion d'épisode, de passage vers un au-delà où s'abolit le temps et s'instaure l'éternité; ou encore, celle du seul présent, de l'existentiel, absurde et imperméable à tout sens? Même les bornes chronologiques empruntent des mesures diverses: celles des phénomènes de longue, de moyenne ou de courte durée, chères à Braudel et à sa représentation des civilisations; celles de la succession des modes de production postulée par le matérialisme historique; celles de phénomènes plus circonscrits, mais passionnants par leur intensité et leur durée relativement brève: la Révolution des Lumières, la Révolution française, la Révolution industrielle - autant d'expressions parmi

\footnotetext{
$8 \quad$ A. Toynbee, L'histoire. Les grands mouvements de l'histoire à travers le temps, les civilisations, les religions (Paris et Bruxelles, 1975), 41.

9 D. Richet, «La place de Fernand Braudel dans l'historiographie d'aujourd'hui», in J. Delumeau et al., L'histoire et ses méthodes (Lille, 1981), 41.

10 F. Braudel, Civilisation matérielle et capitalisme (Paris, 1967), 228.
} 
d'autres qui renvoient à des discontinuités de nature globale, sociétale. Or l'historien puise justement son matériau dans ces halètements inégaux et souvent imprévus des sociétés, non dans le balancement d'un métronome impavide.

L'histoire permettrait donc de dégager des éléments de signification à partir d'événements qualifiés de «faits» ${ }^{11}$, de la constitution de sociétés et de leur évolution. Non des sens de nature immanente, ceux des philosophes, mais des sens concrets-abstraits par lesquels des valeurs et la recherche d'immanence réintègrent l'évolution concrète de civilisations. Elle prend en compte l'action des hommes qui tentent de concilier leurs plans et leurs valeurs avec les réalités géotechniques et de déboucher sur un arbitrage viable entre eux ${ }^{12}$. Elle charrie donc des succès relatifs, limités, sans parler du défi de l'échec et de la mort.

Or c'est peut-être justement ces niveaux qui m'ont le plus intéressé à l'histoire, car ils soudaient des préoccupations philosophiques et littéraires, d'une part, et la réalité vécue, d'autre part. Deux auteurs m'avaient particulièrement frappé par leur saisie de la condition humaine et par les défis qu'ils proposaient: Pascal et Malraux. «L'important, dira le second, c'est de ne pas être vaincu». «Qu'on s'imagine, suggère Pascal, un nombre d'hommes dans les chaînes, et tous condamnés à mort, dont les uns étant chaque jour égorgés à la vue des autres, ceux qui restent voient leur propre condition dans celle de leurs semblables, et, se regardant les uns les autres avec douleur et sans espérance, attendent à leur tour. C'est l'image de la condition des hommes.» (Pensées, Br. 199). D'où sa notion de pari pour un au-delà qui rachète l'absurdité de cette condition, de l'histoire pourrait-on dire. Malraux braque son objectif sur la même condition dans son oeuvre, en particulier dans le roman La Voie royale, alors que le héros blessé, Perken, voit son sort dépendre de la rapidité avec laquelle un interprète indigène traduit ses paroles au chef de la tribu: «Sa destinée, à lui, Perken, se jouait sur cette masse vivante. Sa vie aboutissait comme à un passage à ces jambes couvertes d'eczéma, à ce pagne ignoble et sanglant, cette humanité capable seulement de pièges et de ruse, ainsi que les bêtes de la forêt. Il dépendait totalement de cet être, de ses pensées de larve.» Or la mort, c'est l'échec final: «à partir d'elle rien ne peut être compensé.» Pas tout à fait, cependant: Perken n'a-t-il pas conféré un sens à sa vie et à sa mort en les choisissant préalablement? Et puis, il y a le disciple, Claude, qui perpétuera la pensée du maître à travers le temps et lui assurera une continuité. Et Malraux, que l'histoire fascine au plus haut point, propose finalement un défi, différent sans doute de celui de Pascal, mais de même nature: forger et imposer à l'absurde du destin, un sens qui ne

\footnotetext{
11 Sur cette notion, voir E.H. Carr, What is History (Londres, 1965).

12 Sur ces questions, voir G. Paquet et J.-P. Wallot, «Pour une méso-histoire du XIX siècle canadien», RHAF, 33, 3 (décembre 1979): 389-394, 419-424.
} 
peut venir que de coups de griffe conscients dans la carte de l'histoire ou de la création d'oeuvres d'art qui défient le temps, ordonnent et expriment les civilisations et assurent la mainmise de la conscience sur la fatalité. Comment être indifférent à de telles évocations et de tels appels, vers 1660 , en 1930, 1954 ou 1983 ?

L'histoire, c'est ce témoin, cet éveil de la conscience qui lance un défi à l'effacement et à l'absurdité qui nous attendent. Comme elle doit composer avec des «faits», elle s'avère à la fois plus frustrante et plus satisfaisante que la fiction littéraire. Elle rend compte d'états de faits parfois imprévisibles ou qui dépassent le «naturel», voire le plausible. «L'histoire, écrivais-je récemment, intéresse parce que l'homme, hors de la littérature, sort du «naturel»: il pense l'ordre naturel, le contredit, l'ordonne, l'apprivoise, l'exploite. Le bon écrivain invente. L'homme historique, seul ou en société, «surprend»... Voilà la source de l'engouement pour l'histoire: nul écrivain n'eût pu inventer Hitler!» ${ }^{13}$ Mais en histoire, comme dans les arts, une autre intuition de Malraux sonne juste: L'historien décode le passé à partir de postulats et de l'examen de sources, mais aussi de la lecture qu'en ont faite les historiens antérieurs, tout comme l'artiste peint peut-être moins le réel tel qu'il le voit que le réel lu à travers des générations de peintres antérieurs. De sorte qu'aux sources et aux questions du présent, il faut ajouter une troisième dimension, celle qu'on appelle l'historiographie.

J'ai dit au début que l'histoire me refoulait vers l'universel. C'est que dans cette discipline, rien n'est plus universel que le singulier ni plus singulier que l'universel. Il est vrai qu'aujourd'hui le passé se conjugue mal au singulier et qu'on lui préfère les pluriels: les groupes sociaux, les forces sociales, les nations et les régions, les phénomènes de masse, les collectifs de toute nature. Ces perspectives et ces méthodes nouvelles visent toutefois à optimiser en quelque sorte les résultats de l'opération essentielle de l'historien: expliquer ce qui est arrivé et pourquoi, à partir de vestiges presque toujours épars et incomplets, trop bavards ou trop silencieux selon les divers niveaux de réalité, presque toujours muets sur ces gestes répétés, acquis, transmis, qui imprègnent la vie quotidienne. Il faudrait, selon l'expression du romancier américain John Updyke, reconstruire la tempête de neige à partir d'un flocon qui fond dans la main. L'histoire est donc oeuvre d'intelligence, de conscience et de passion, car elle touche à la fois à nos origines, à la condition humaine et à la réinvention d'un monde plausible à partir de notations très incomplètes qui jonchent la partition du temps.

$$
\text { ** }
$$

Pour conclure, je dois avouer que l'histoire est bien une «science de la vie» (Lucien Febvre): une science qui intéresse parce que «le passé

13 J.-P. Wallot, «De l'indiscipline...», 59. 
contient, en préformation, le présent» (Pierre-Henri Simon) et que la conscience du passé aide à déblayer les valeurs du présent. Elle est une science et un art qui expriment les inquiétudes et les pratiques des hommes porteurs d'espoirs et de desseins sur l'avenir, qu'elle tourne rétrospectivement comme pour en pister l'origine et en jauger la plausibilité dans les replis du passé, comme pour en extraire la clé du déroulement ultérieur. Par le miroir de l'histoire, où le présent n'est que le vif-argent qui suscite l'interrogation, l'homme projette son destin et sa signification dans son passé pour penser son avenir. Il prédit rétrospectivement. Il faut des rétrodictions qu'il alimente à l'angoisse indomptée de son destin qu'il veut mieux comprendre et assumer.

Les disciplines diffèrent de l'une à l'autre. Pourtant, l'indiscipline s'imipose. Il faut déborder les frontières et se tourner vers l'universel, à défaut de se trouver «d'avance vieilli» (Pierre Vilar) ${ }^{14}$. En effet, le poète, l'artiste, le philosophe, l'historien, que cherchent-ils sinon la conscience de ce que nous avons été, de ce que nous sommes et de ce que nous pouvons devenir, et leur expression adéquate. Or toute prise de conscience est fugace, mouvante, trompeuse comme ces alluvions récentes qui recouvrent les dépôts passés. Elle est inégale et n'est jamais achevée. Elle baigne dans l'incertitude d'un mouvement dont on ne peut tout au plus qu'esquisser un éventail de trajectoires plausibles, sans vraiment être sûr de l'aboutissement final. Voilà la leçon de vingt ans de pratique de l'histoire: l'absence de certitude, de point de chute définitif; la nécessité de pousser toujours plus loin, de combler les vides, ou, comme dit Pierre-Henri Simon dans un commentaire sur Malraux: «Nous acceptons de prendre la route au point où les générations précédentes l'ont amenée, et de la frayer à notre tour, de nos mains douloureuses, sans autre certitude que la joie de chaque obstacle surmonté, de chaque progrès... accompli vers le terme inconnu de notre marche dans le temps.» ${ }^{15}$ C'est notre destin d'hommes. C'est celui de l'historien aussi, voire l'essence même de sa discipline.

\footnotetext{
${ }_{14} \mathrm{P}$. Vilar, Une histoire en construction. Approche marxiste et problématiques conjoncturelles (Paris, 1982), 425.

15 P.-H. Simon, «André Malraux», in Le Levoir, 16 novembre 1957.
} 